\section{$50 \%$ duty cycle may be inappropriate to achieve a sufficient chest compression depth when cardiopulmonary resuscitation is performed by female or light rescuers}

\author{
Chang Jae Lee, Tae Nyoung Chung, Jinkun Bae, Eui Chung Kim, \\ Sung Wook Choi, Ok Jun Kim \\ Department of Emergency Medicine, CHA University School of Medicine, Seongnam, Korea
}

Objective Current guidelines for cardiopulmonary resuscitation recommend chest compressions (CC) during 50\% of the duty cycle (DC) in part because of the ease with which individuals may learn to achieve it with practice. However, no consideration has been given to a possible interaction between $D C$ and depth of $C C$, which has been the subject of recent study. Our aim was to determine if 50\% DC is inappropriate to achieve sufficient chest compression depth for female and light rescuers.

Methods Previously collected CC data, performed by senior medical students guided by metronome sounds with various down-stroke patterns and rates, were included in the analysis. Multiple linear regression analysis was performed to determine the association between average compression depth (ACD) with average compression rate (ACR), DC, and physical characteristics of the performers. Expected ACD was calculated for various settings.

Results DC, ACR, body weight, male sex, and self-assessed physical strength were significantly associated with ACD in multivariate analysis. Based on our calculations, with $50 \%$ of DC, only men with ACR of 140/min or faster or body weight over $74 \mathrm{~kg}$ with ACR of 120/min can achieve sufficient ACD.

Conclusion A shorter DC is independently correlated with deeper CC during simulated cardiopulmonary resuscitation. The optimal DC recommended in current guidelines may be inappropriate for achieving sufficient $C D$, especially for female or lighter-weight rescuers.

Keywords Cardiopulmonary resuscitation; Clinical competence; Education; Prognosis; Heart arrest

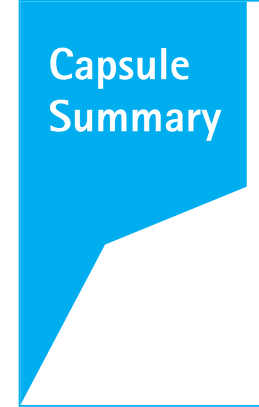

What is already known

A duty cycle of $50 \%$ is recommended for chest compressions during cardiopulmonary resuscitation due to its ease of achievement with training.

What is new in the current study

Current recommendations for duty cycle may be inappropriate to achieve a sufficient chest compression depth especially for female or light rescuers.
elSSN: 2383-4625

Received: 30 October 2014

Revised: 5 December 2014

Accepted: 14 December 2014

Correspondence to: Tae Nyoung Chung Department of Emergency Medicine, CHA University School of Medicine, 59 Yatap-ro, Bundang-gu, Seongnam 463-712, Korea

E-mail: hendrix74@gmail.com

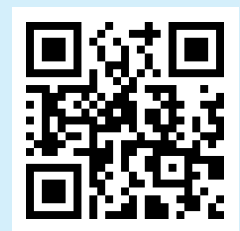

How to cite this article: Lee CJ, Chung TN, Bae J, Kim EC, Choi SW, Kim 0 J. $50 \%$ duty cycle may be inappropriate to achieve a sufficient chest compression depth when cardiopulmonary resuscitation is performed by female or light rescuer. Clin Exp Emerg Med 2015;2(1):9-15.

This is an Open Access article distributed under the terms of the Creative Commons Attribution Non-Commercial License (http:// creativecommons.org/licenses/by-nc/3.0/). 


\section{INTRODUCTION}

In cardiopulmonary resuscitation (CPR), achievement and maintenance of sufficient depth of chest compressions is critical for the survival of cardiac arrest victims. ${ }^{1-5}$ Various efforts have been attempted to identify efficient means of maintaining sufficient chest compression depth. ${ }^{6-10}$ Current CPR guidelines emphasize not only the depth, but also other technical components of chest compression, such as rate, recoil property, interruption time, and duty cycle (DC), in terms of achieving and maintaining good quality. ${ }^{11}$ The term "duty cycle" means the proportion of the time spent compressing the chest from the start of 1 cycle of compression and the start of the next. Although DCs ranging from 20\% to $50 \%$ can result in adequate coronary and cerebral perfusion, ${ }^{12-15}$ a DC of 50\% is recommended in the current guidelines because it is easy to achieve with practice. ${ }^{11}$ However, this recommendation does not consider possible interactions of the DC with other chest compression quality factors, such as the depth and rate, as has been studied elsewhere. ${ }^{16}$ Moreover, a recent study has shown that a faster down-stroke, which is thought to induce a shorter DC, correlates with a deeper compression depth during metronome-guided CPR. ${ }^{17}$ Baseline data of volunteers who participated in the study suggested a correlation between physical characteristics, such as sex, height, and weight, with the depth of chest compressions; further, there may be a possible interaction among the physical characteristics, DC, and depth. If so, the tendency of deeper compression with lower $\mathrm{DC}$, as shown in the results, might differ between rescuers with different physical characteristics. Current recommendations for a 50\% DC may be inappropriate for induction of chest compressions with sufficient depth for certain rescuers. We therefore aimed to assess true correlation of DC with the depth of chest compressions considering the interaction of physical characteristics, and determine if 50\% DC is inappropriate to achieve sufficient chest compression depth in certain conditions, using the regression equation built from a previously collected data set.

\section{METHODS}

Three hundred twenty-four compression-only CPR performance data sets, independently conducted by 27 volunteers, were included in the analysis. ${ }^{17}$ This prospective, randomized, cross-over trial was performed under the approval of our institutional review board. Senior medical students who had finished two sessions of regular CPR training courses based on the American Heart Association Basic Life Support course within the past year, performed 12 sets of continuous chest compressions for 1.5 minutes ( $30 \mathrm{sec}-$ onds for practice and 1 minute for measurement) following the prepared metronome guidance in a random sequence with three down-stroke patterns (normal, fast, and very fast), and four rates $(80,100,120$, and 140 compressions/min), based on a random number table generated by Microsoft Office Excel 2007 (Micro-

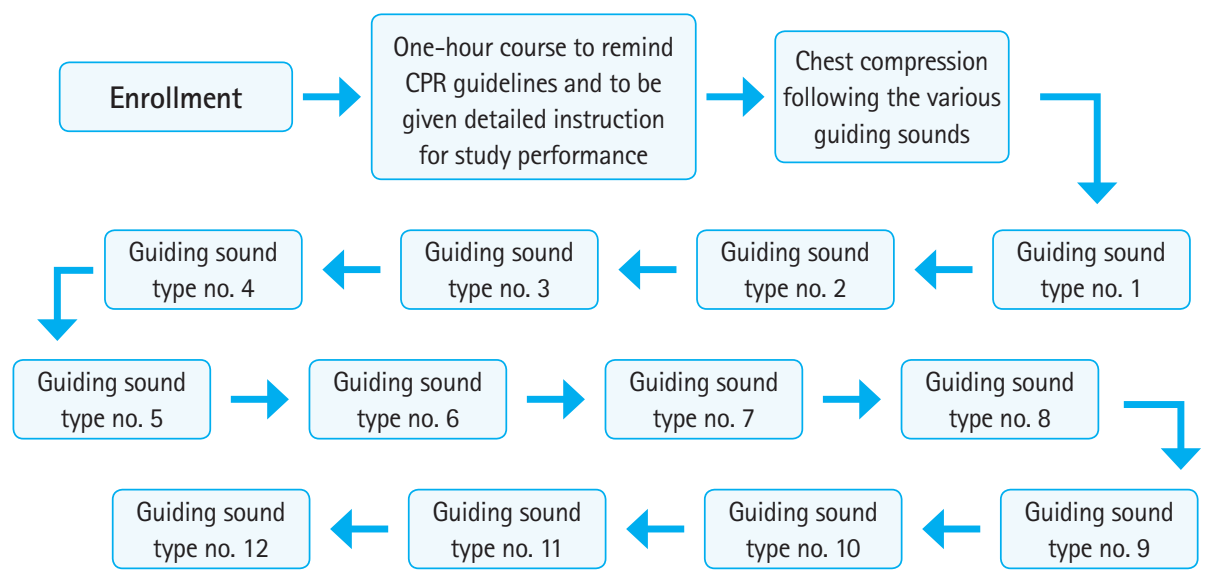

Preparation of guiding sounds: two beep sounds one for compression, and the other for release Variation in the ratios of the interval between the first and the second beeps to induce various duty cycles 12 types of guiding sounds consisted of 3 ratio patterns $(1,0.5,0.3)$ for 4 compression rates $(80,100,120,140)$

Fig. 1. Flow diagram of study performances. The participants performed 12 sets of continuous chest compressions for 1.5 minutes (30 seconds for practice and 1 minute for measurement) following the prepared metronome guidance in a random sequence. To avoid the effect of rescuer fatigue, sufficient rest time was allowed between the trials (more than 60 minutes), and the maximum number of performances in a day was limited to 4 . CPR, cardiopulmonary resuscitation. 
soft Corporation, Redmond, WA, USA) (Fig. 1). A Resusci Anne manikin with a PC skill-reporting system (Laerdal, Stavanger, Norway) was used to measure and record CPR data. Chest compression guiding sounds were synthesized using Reason audio synthesis software (Propellerhead, Stockholm, Sweden) with the Waves gold bundle signal processing software package (Waves, Knoxville, TN, USA). Participant baseline data such as sex, age, body weight, and height measurements were collected, and physical strength was gauged through a self-assessment that used a 10-point visual analogue scale. Average compression depth (ACD, mm), average compression rate (ACR, numbers/min), DC (\%), and proportion of chest compressions with incomplete release and incorrect hand position (\%) were all measured and recorded using the PC skill-reporting system.

Every performance was analyzed to assess the effect of DC, $A C R$, baseline data, and self-assessed physical strength on ACD. Analysis of covariance (ANCOVA) was performed to detect differences in ACD by rescuer sex, while considering the effect of other covariates that were significant on univariate analysis. Multiple linear regression analysis was performed, including every parameter showing significant result in ANCOVA as an independent variable and $A C D$ as a dependent one. Expected $A C D$ values of performances with $50 \% \mathrm{DC}$, by compression rate, were calculated for each sex based on median body weight and self-assessed physical strength. The same calculation was performed according to the quartile values of weight in each sex.

Mean and standard deviation values were used to report the data with a normal distribution, and a median with interquartile range was used for the nonparametric variables. The results of the statistical tests were considered significant when the P-value was less than 0.05. Microsoft Office Excel 2010 (Microsoft Cor- poration) was used to record and analyze the data, and IBM SPSS Statistics ver. 21.0 (IBM Corp., Armonk, NY, USA) was used for statistical calculations.

\section{RESULTS}

Participant baseline data are summarized in Table 1. The distribution of participant body weight is described in Fig. 2. There was a significant difference in mean value of ACD between female and male rescuers (Fig. 3A). Not only DC and ACR, which were major determinant of compression depth in previous works, ${ }_{1}^{16,17}$ but also height, weight, and self-assessed physical strength of rescuer, which showed significant correlation with ACD in newly performed analysis, were included in ANCOVA as covariates (Fig. 4). ANCOVA revealed that sex and all covariates were correlated with ACD except the height of rescuer (P-values of self-assessed physical strength and height $=0.010,0.794$, respectively, all other $<0.001$ ) (Fig. 3B).

Table 1. Baseline data of the participants

\begin{tabular}{|c|c|c|c|c|}
\hline & \multirow[t]{2}{*}{ Overall } & \multicolumn{2}{|c|}{$\begin{array}{l}\text { Average compression } \\
\text { depth }(\mathrm{cm})\end{array}$} & \multirow[t]{2}{*}{ P-value } \\
\hline & & $\geq 5$ & $<5$ & \\
\hline Age (yr) & $27(26-29)$ & $28(26-29)$ & $27(26-29)$ & $0.097^{\mathrm{a}}$ \\
\hline Body weight $(\mathrm{kg})$ & $64.3 \pm 11.3$ & $69.3 \pm 8.2$ & $58.3 \pm 11.5$ & $<0.001^{\text {b) }}$ \\
\hline Height (cm) & $170.7 \pm 7.7$ & $173.9 \pm 6.4$ & $167.0 \pm 7.6$ & $<0.001^{\text {b) }}$ \\
\hline Male sex $(\%)$ & 66.7 & 89.8 & 38.8 & $<0.001^{\mathrm{c}}$ \\
\hline Self-assessed physical strength & $7(7-8)$ & $8(7-8)$ & $7(6-8)$ & $<0.001^{\text {a) }}$ \\
\hline
\end{tabular}

The format of the numeric values are presented as median (interquartile range) for age and self-assessed fitness or mean \pm standard deviation for body weight and height.

${ }^{a}$ Result from t-test. ${ }^{b}$ Result from Mann-Whitney U-test. ${ }^{\text {c }}$ Result from chi-square test.
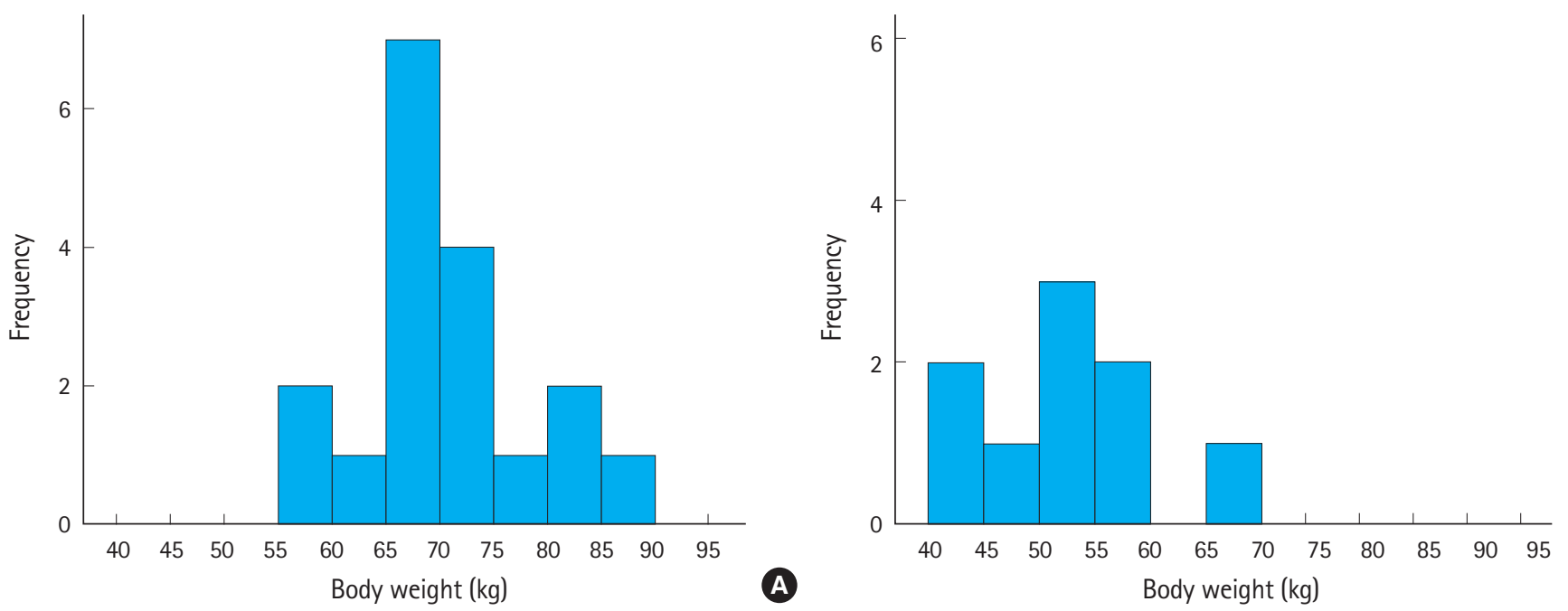

Fig. 2. Distribution of participant body weight by sex. (A) Male and (B) female. 

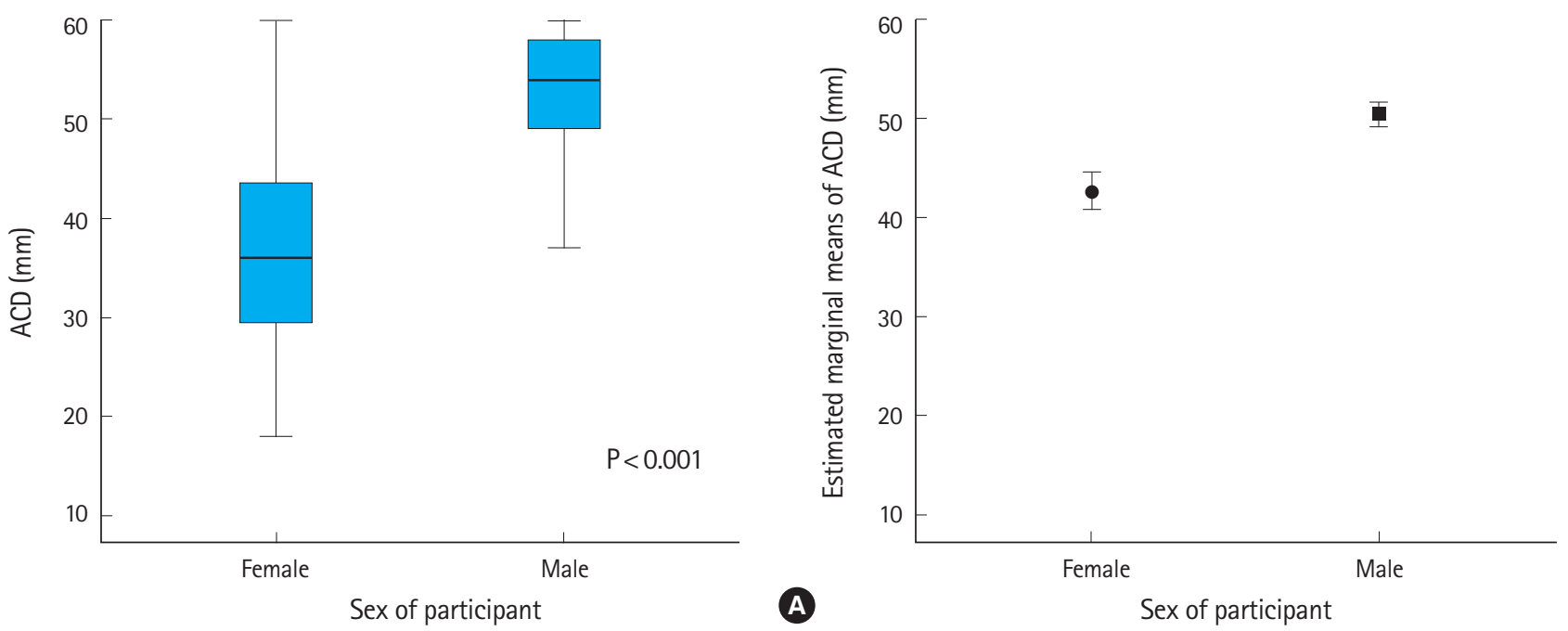

B

Fig. 3. Depth of chest compression by rescuer sex. (A) Distribution of average compression depth (ACD) according to the sex of participants. The P-value was calculated from the Mann-Whitney U-test. (B) Estimated marginal means of ACD according to the sex of participants, which were calculated from analysis of covariance.
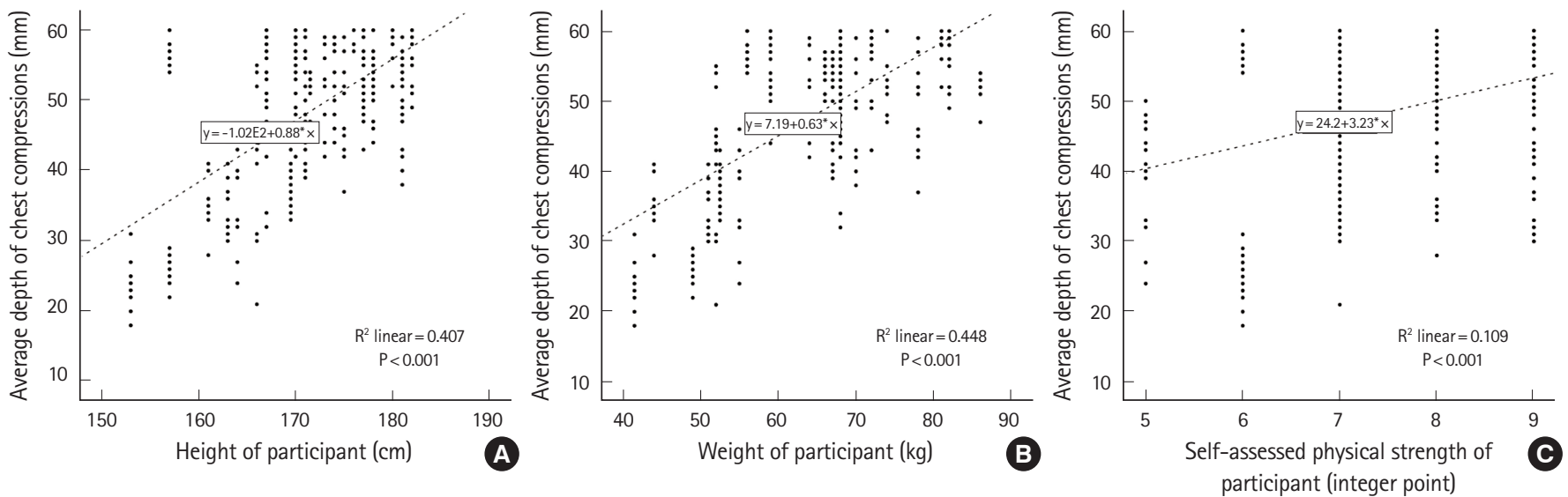

Fig. 4. Correlation of the average compression depth with (A) height of the participant, (B) weight of the participant, and (C) self-assessed physical strength of the participant. Line, equation, and P-values were calculated from simple linear regression analysis.

Table 2. Results of the multiple linear regression analysis to determine the factors contributing to the average depth of chest compression

\begin{tabular}{lcccc}
\hline Variable & Regression coefficient & Standard error & t & P-value \\
\hline Intercept & 20.090 & 4.583 & 4.384 & $<0.001$ \\
Duty cycle (\%) & -0.399 & 0.055 & -7.281 & $<0.001$ \\
Average compression rate (number/min) & 0.073 & 0.018 & 4.052 & $<0.001$ \\
Body weight (kg) & 0.358 & 0.052 & 6.879 & $<0.001$ \\
Male sex (dichotomous: yes, $1 ;$ no, 0) & 8.016 & 1.290 & $<.216$ \\
Self-assessed physical strength (10 integer points) & 1.048 & 0.371 & 2.826 & 0.001 \\
\hline
\end{tabular}

DC, ACR, body weight, male sex, and self-assessed physical strength were significantly correlated with ACD on multiple linear regression analysis (adjusted $R^{2}=0.595, P<0.001$ ) (Table 2). Expected ACDs according to ACR and sex calculated from the regression equation are shown in Table 3. Similar calculations according to the quartiles of weight and sex of the rescuers are shown in Table 4. 
Table 3. Expected values of average compression depth $(\mathrm{mm})$ with the duty cycle of 50\%, according to the rate of chest compressions and the sex of rescuer, calculated from the regression equation

\begin{tabular}{lcc}
\hline $\begin{array}{l}\text { Rate of chest compression } \\
\text { (compression/min) }\end{array}$ & Male & Female \\
\hline 100 & 47.7 & 33.4 \\
110 & 48.4 & 34.1 \\
120 & 49.1 & 34.9 \\
130 & 49.9 & 35.6 \\
140 & 50.6 & 36.3 \\
\hline
\end{tabular}

Regression equation: average compression depth $=-0.399 \times$ [duty cycle] $+0.073 \times$ [average compression rate] $+0.358 \times[$ body weight] $+8.016 \times$ [male sex $]+1.048 \times$ [self assessed physical strength]+20.090. Body weight used in the calculation: 68 for male and 52 for female. Self assessed physical strength used in the calculation: 7.5 for male and 7 for female. Duty cycle used for calculation: 50\% in both sexes.

\section{DISCUSSION}

This is the first study to assess DC of chest compression and other components that may interact with each other during CPR. Results suggest that a shorter DC is an independent predictor of deeper chest compression and imply that the 50\% DC recommended in current CPR guidelines may be inappropriate.

Participant baseline data suggest the possibility of an association between depth of chest compression and rescuer body weight, height, sex, and self-assessed physical strength. Body weight, height, and self-assessed physical strength showed linear association with $A C D$ in simple regression analysis. A possible sex effect on ACD was supported by the result of ANCOVA, which confirmed that the difference in ACD was caused by the rescuer's sex.

As shown in our previous work, a shorter DC, which was interpreted as a shorter compression-phase, is correlated with a deeper chest compression in a linear manner, regardless of the speed of chest compressions. ${ }^{17}$ Results of the multiple linear regression analysis confirmed that a shorter DC is an independent predictor of deeper chest compressions, which means that a shorter DC is correlated with a deeper compression regardless of the sex or body weight of the rescuer. Moreover, the direct correlation between ACR and ACD supported the results of Chung et al., ${ }^{16}$ which showed that a higher compression rate is associated with deeper chest compression. We also identified a significant difference in ACD based on sex; male rescuers achieved a significantly greater $A C D$ than female rescuers. This finding is consistent with that of Peberdy et al., ${ }_{1}^{18}$ which showed that the sex and age of the caregiver affect chest compression depth. Age was not a statistically significant variable in our study, even on simple comparison. This discordance may be due to the narrow age range of our participants, as we enrolled only students from one class in our medical school.
Table 4. Expected values of average compression depth $(\mathrm{mm})$ with the duty cycle of 50\% and the average compression rate of $120 / \mathrm{min}$, according to the quartiles of body weight in each sex of rescuer, calculated from the regression equation

\begin{tabular}{lcc}
\hline Quartile & Male & Female \\
\hline Quartile 1 & 48.4 & 33.8 \\
Quartile 2 & 49.1 & 34.9 \\
Quartile 3 & 51.3 & 35.9
\end{tabular}

Regression equation: average compression depth $=-0.399 \times$ [duty cycle] $+0.073 \times$ [average compression rate] $+0.358 \times$ [body weight] $+8.016 \times$ [male sex $]+1.048 \times$ [self assessed physical strength]+20.090. Quartile values used in the calculation: quartile 1, 66 for male and 49 for female; quartile 2, 68 for male and 52 for female; quartile 3, 74 for male and 55 for female. Body weight used in the calculation: 68 for male and 52 for female. Self assessed physical strength used in the calculation: 7.5 for male and 7 for female. Duty cycle used for calculation: 50\% in both sexes.

The association between self-assessed physical strength and ACD is also an interesting result. However, participant baseline characteristics should not be generalized as factors that directly influenced the quality of chest compressions (although they were statistically significant on multivariate analysis), solely based on our results. Rather, they would be better treated as covariates correcting the effect of the factors that were directly assessed during every independent performance, including $D C$ and $A C R$, because the baseline data were used repeatedly in the analysis. Further study is necessary to investigate the actual association of self-reported physical strength with the depth of chest compressions.

The independent inverse correlation between DC and ACD observed in our study may be somewhat contrary to the results of previous studies, which suggested that longer DC induces increases in aortic pressure and cerebral blood flow. ${ }^{13,19}$ However, it should also be noted that DC increased, while the force of chest compression was fixed in both studies; this did not allow us to analyze the interaction between the DC and compression force. Considering this information, our finding of a correlation between $\mathrm{DC}$ and $A C D$ may suggest an interacting effect of $D C$ on chest compression force.

Expected ACDs calculated from the regression equation provide one of the most important findings: 50\% DC, as recommended in the current CPR guidelines, may not be appropriate to achieve sufficient chest compression depth, especially for female and lighter-weight rescuers. Furthermore, some guidelines recommend an ACR ranging between 100/min and 120/min and a DC of 50\%; however, considering these values, our expected ACDs were below the recommended depth of chest compressions. The expectation of sub-optimal chest compression depth with 50\% DC even with faster rates, which were known to enhance $A C D$ in previous 
studies, $^{16,17}$ implies the necessity for a shorter DC than the current recommendation. Calculation of results according to rescuer weight quartiles indicated that expected ACD for only male rescuers with body weights of quartile 3 (75 percentile) are in the recommended range, which also supports the inappropriateness of the recommended 50\% DC. This calculation result suggests that the currently recommended DC may be generally inappropriate for light rescuers, considering that the multivariate analysis identified the body weight of the participant as an independent predictor of chest compression depth. Although our results were from a crossover trial, because of which the specific results obtained from the baseline data of the participants should not be directly accepted (as mentioned above), their strong association with ACD might reflect the trend. Hence, the recommendation of $50 \%$ DC should be reconsidered at least for female or lighterweight rescuers.

Our study had a few limitations. First, the study was a reanalysis of previously collected data derived from simulated CPR performances. Subsequently, direct implementation of our findings to a clinical setting should be performed with great caution. Furthermore, baseline data of the participants were used repetitively in the multivariate analysis, because the datasets were obtained from cross-over trials conducted by a relatively small number of participants. Hence, it might cause some deviation of the results, even if the performances were conducted in a fully independent manner. Second, every chest compression in the study was performed with audio tone guiding because of the need to induce a certain DC range. Therefore, there could have been an additive effect of metronome guidance on the chest compression quality. Third, baseline fitness of the participants was measured by a selfreported scale, which was not an objective method and might cause some flaws in the results.

In conclusion, shorter DC is independently correlated with deeper chest compression during simulated CPR and the recommended DC in the current guidelines may be inappropriate for achieving sufficient compression depth, especially for female or lighterweight rescuers.

\section{CONFLICT OF INTEREST}

No potential conflict of interest relevant to this article was reported.

\section{REFERENCES}

1. Edelson DP, Abella BS, Kramer-Johansen J, et al. Effects of compression depth and pre-shock pauses predict defibrilla- tion failure during cardiac arrest. Resuscitation 2006;71:13745.

2. Gallagher EJ, Lombardi G, Gennis P. Effectiveness of bystander cardiopulmonary resuscitation and survival following out-ofhospital cardiac arrest. JAMA 1995;274:1922-5.

3. Kramer-Johansen J, Myklebust $H$, Wik L, et al. Quality of outof-hospital cardiopulmonary resuscitation with real time automated feedback: a prospective interventional study. Resuscitation 2006;71:283-92.

4. Van Hoeyweghen RJ, Bossaert LL, Mullie A, et al. Quality and efficiency of bystander CPR: Belgian Cerebral Resuscitation Study Group. Resuscitation 1993;26:47-52.

5. Wik L, Steen PA, Bircher NG. Quality of bystander cardiopulmonary resuscitation influences outcome after prehospital cardiac arrest. Resuscitation 1994;28:195-203.

6. Cloete $G$, Dellimore KH, Scheffer C, Smuts MS, Wallis LA. The impact of backboard size and orientation on sternum-to-spine compression depth and compression stiffness in a manikin study of CPR using two mattress types. Resuscitation 2011; 82:1064-70.

7. Handley AJ, Handley SA. Improving CPR performance using an audible feedback system suitable for incorporation into an automated external defibrillator. Resuscitation 2003;57:5762.

8. Mirza M, Brown TB, Saini D, et al. Instructions to "push as hard as you can" improve average chest compression depth in dispatcher-assisted cardiopulmonary resuscitation. Resuscitation 2008;79:97-102.

9. Pozner CN, Almozlino A, Elmer J, Poole S, McNamara D, Barash D. Cardiopulmonary resuscitation feedback improves the quality of chest compression provided by hospital health care professionals. Am J Emerg Med 2011;29:618-25.

10. Skorning M, Beckers SK, Brokmann JCh, et al. New visual feedback device improves performance of chest compressions by professionals in simulated cardiac arrest. Resuscitation 2010; 81:53-8.

11. Berg RA, Hemphill R, Abella BS, et al. Part 5: adult basic life support: 2010 American Heart Association Guidelines for Cardiopulmonary Resuscitation and Emergency Cardiovascular Care. Circulation 2010;122(18 Suppl 3):S685-705.

12. Feneley MP, Maier GW, Kern KB, et al. Influence of compression rate on initial success of resuscitation and 24 hour survival after prolonged manual cardiopulmonary resuscitation in dogs. Circulation 1988;77:240-50.

13. Halperin HR, Tsitlik JE, Guerci AD, et al. Determinants of blood flow to vital organs during cardiopulmonary resuscitation in dogs. Circulation 1986;73:539-50. 
14. Handley AJ, Handley JA. The relationship between rate of chest compression and compression:relaxation ratio. Resuscitation 1995;30:237-41.

15. Maier GW, Tyson GS Jr, Olsen CO, et al. The physiology of external cardiac massage: high-impulse cardiopulmonary resuscitation. Circulation 1984;70:86-101.

16. Chung TN, Kim SW, You JS, Cho YS, Chung SP, Park I. A higher chest compression rate may be necessary for metronome-guided cardiopulmonary resuscitation. Am J Emerg Med 2012;30: 226-30.
17. Chung TN, Bae J, Kim EC, et al. Induction of a shorter compression phase is correlated with a deeper chest compression during metronome-guided cardiopulmonary resuscitation: a manikin study. Emerg Med J 2013;30:551-4.

18. Peberdy MA, Silver A, Ornato JP. Effect of caregiver gender, age, and feedback prompts on chest compression rate and depth. Resuscitation 2009;80:1169-74.

19. Babbs CF, Thelander K. Theoretically optimal duty cycles for chest and abdominal compression during external cardiopulmonary resuscitation. Acad Emerg Med 1995;2:698-707. 